\title{
ORIGINAL
}

\section{INCIDENCIA Y FACTORES DE RIESGO DE LAS INFECCIONES \\ QUIRÚRGICAS DE BY-PASS VASCULARES EN EL HOSPITAL JUAN CANALEJO DE A CORUÑA EN EL PERÍODO 2000-2002}

\author{
Miguel Rosales Rodríguez (1), Teresa Jiménez Martínez (1), Vicente Domínguez Hernández (1), \\ Juan Ramón Segura Iglesias (2) Eduardo Díaz Vidal (2) \\ (1) Servicio de Medicina Preventiva y Salud Publica. Complejo Hospitalario Universitario Juan Canalejo. A Coruña \\ (2) Servicio de Cirugía Vascular. Complejo Hospitalario Universitario Juan Canalejo. A Coruña
}

\section{RESUMEN}

Fundamento: La cirugía vascular de by pass se utiliza cada vez con mayor frecuencia en sujetos de edad avanzada y con varios factores de riesgo. La infección quirúrgica de estas intervenciones es una complicación devastadora que se asocia con unas altas tasas de una complicación devastadora que se asocia con unas altas tasas de
morbilidad y mortalidad. Los objetivos del estudio son conocer la incidencia de infecciones quirúrgicas en pacientes sometidos a by pass con prótesis vasculares, los factores de riesgo asociados a ellas y el tipo de prótesis que presenta mayor tasa de infección.

Métodos: Se llevó a cabo un estudio cohortes retrospectivo en el Servicio de Cirugía Vascular del Hospital Universitario Juan Canalejo, realizado durante los años 2000-2002. Los criterios de infección aplicados fueron los del Center for Disease Control. Las medidas de frecuencia calculadas fueron incidencia acumulada y el riesgo relatifrecuencia calculadas fueron incidencia acumulada y el riesgo relativo como medida de asociación. Las pruebas estadísticas empleadas
fueron el Chi cuadrado y el Chi cuadrado de tendencias, T de Student para análisis univariado y la regresión logística para el multivariado.

Resultados: La tasa de incidencia acumulada entre los años 2002-2002 fue de $22,6 \%$ a $12,1 \%$. Los microorganismos más frecuentes fueron Staphilococcus aureus, Staphilococcus aureus meticilina resistente, Staphilococcus epidermidis, y Escherichia coli Los factores de riesgo relacionados con la infección encontrados fueron factores de riesgo relacionados con la infección encontrados fueron
el sexo femenino $(\mathrm{RR}=1,8)$ riesgo anestésico $>2(\mathrm{R}=1,7)$ y la el sexo femenino $(\mathrm{RR}=1,8)$ riesgo anestésico $>2(\mathrm{RR}=1,7)$ y la
implantación de prótesis venosa de vena safena del paciente $(\mathrm{RR}=$ $3,8)$

Conclusiones: Los factores de riesgo identificados han sido el sexo femenino, el riesgo anestésico y el tipo de prótesis utilizada. Todos ellos condicionan la complicación infecciosa en la cirugía de by-pass.

Palabras clave: Hospital. Procedimientos quirúrgicos vasculares. Infección de herida operatoria. España.

Correspondencia:

Miguel Rosales Rodríguez

Complejo Hospitalario Universitario Juan Canalejo

As Xubias de Arriba 84

1506 A Coruña

\section{ABSTRACT}

Vascular By-pass Surgery Infection Incidence and Risk Factors at the Juan Canalejo Hospital in Corunna within the 2000-2002 Period

Background: Vascular by-pass surgery is being used increasingly more frequently on elderly patients entailing several risk factors. Surgical infections in these procedures are a devastating complication which is related to high morbility and death rates. This study is aimed at ascertaining the incidence of surgical infections among patients having undergone a by-pass procedure with vascular prostheses, the risk factors associated thereto and the type or prosthesis entailing a lower infection rate.

Methods: A retrospective cohort study was conducted at the Vascular Surgery Unit of the Juan Canalejo University Hospital throughout the 200-2002 period. The Disease Control Center infection criteria were those applied. The frequency measurements calculated were cumulative incidence and relative risk as an association measurement. The statistical tests employed were the Chi-square an the Chi-square trend tests, the Student's-T for univariate analysis and logic regression for the multi-variate analysis.

Results: The cumulative incidence rate for the 2000-2002 period was $22.6 \%$ to $12 . \%$. The most frequent microorganisms were Staphylococcus aureus, meticilin-resistant Staphylococcus aureus, Staphylococcus epidermidis, and Escherichia coli. The risk factors related to infection found were female sex $(\mathrm{RR}=1.8)$ anesthetic risk $>2(\mathrm{RR}=1.7)$ and patient implanted with sapheneous vein vascular prosthesis $(\mathrm{RR}=3.8)$.

Conclusions: The risk factors identified were the female sex, anesthetic risk and the type of prosthesis used, all of which condition infection-related complication in by-pass surgery.

Key words: Hospitals. Vascular Surgical Procedures. Surgical Wound Infection. Blood Vessel Prosthesis. Spain. 


\section{INTRODUCCIÓN}

La cirugía vascular de by-pass se emplea cada vez con mayor frecuencia en sujetos de edad avanzada y con varios factores de riesgo, lo que propicia un aumento en la aparición de complicaciones en estos pacientes. Entre ellas las infecciones de heridas quirúrgicas son las más frecuentes. Así la infección de by-pass arterial es una complicación devastadora que se asocia con elevadas tasas de mortalidad y pérdida de miembros ${ }^{1}$.

La incidencia de las infecciones quirúrgicas de by-pass vasculares ha ido declinando a lo largo de los años como resultado de mejora de las técnicas quirúrgicas, de los materiales empleados y de la profilaxis antimicrobiana. No obstante aunque las infecciones son complicaciones relativamente infrecuentes conllevan una elevada morbilidad y mortalidad.

La incidencia de las mismas varía dependiendo de los diferentes estudios, del tipo de infección considerada, del lugar de la incisión, etcétera, oscilando dentro de un rango del $7 \%$ al $44 \%^{2}$ si consideramos todas las infecciones, o entre el $0,5 \%$ a $5 \%$ si sólo tenemos en cuenta las infecciones protésicas propiamente dichas ${ }^{3,4}$; y ello, a pesar de la profilaxis antibiótica utilizada en este tipo de procedimientos ${ }^{5}$.

Una de las razones esgrimidas para estas importantes tasas es el lugar de la incisión. La región inguinal es asiento frecuente de la infección debido a determinados factores entre los cuales destacan la proximidad del área perineal o la presencia de infecciones próximas $^{6}$.

La utilización de diferentes clases de prótesis (fibras de Dacron, homoinjertos o injertos criopreservados) también ha sido objeto de atención por diferentes autores para conocer cual de ellas presenta menores tasas de infección 7 .
El objetivo de nuestro estudio es conocer la incidencia de infecciones quirúrgicas en pacientes sometidos a cirugía de by-pass con prótesis vasculares, así como identificar los potenciales factores de riesgo de los mismos y que tipo de prótesis presenta mayor tasa de infección.

\section{MATERIAL Y MÉTODOS}

El estudio se desarrolló en el servicio de cirugía vascular del complejo hospitalario Juan Canalejo de A Coruña, que cuenta con 29 camas y atiende a la población de referencia del área sanitaria de A Coruña que es de 800.000 habitantes.

Desde enero de 2000 hasta finales de diciembre de 2002 se llevó a cabo un estudio epidemiológico observacional retrospectivo. La cohorte estaba formada por todos los pacientes que tras su alta tenían el código relativo a by-pass mediante cirugía mayor, no incluyéndose los by pass de fístulas para pacientes en diálisis. La revisión de las personas que componían la cohorte se llevó a cabo mensualmente, emitiendo posteriormente el informe correspondiente al servicio implicado.

Recogida de datos: Se revisaron las historias clínicas de todos los pacientes intervenidos por by-pass y mediante la utilización de un registro estandarizado se recogieron las siguientes variables por cada paciente; edad, sexo, diagnóstico, profilaxis quirúrgica, riesgo anestésico (valorado según la Asociación de Anestesia Americana) ASA $^{8}$, duración de la intervención, tipo de prótesis empleada, presencia de diabetes, drenajes, tipo de intervención quirúrgica según la clasificación de Altemeier ${ }^{9}$, intervención urgente o no, y estancia preoperatoria. El índice de riesgo NNIS (National Nosocomial Infection Study) fue calculado basándonos en el percentil 75 de la duración de la intervención, el riesgo anestésico y la clasificación de la intervención quirúrgica, tal y 
Tabla 1

Características de los pacientes estudiados

\begin{tabular}{|c|c|c|}
\hline Variables & Número & Porcentaje \\
\hline $\begin{array}{c}\text { Sexo } \\
\text { Varón } \\
\text { Mujer }\end{array}$ & $\begin{array}{c}534 \\
84\end{array}$ & $\begin{array}{l}86,4 \\
13,6\end{array}$ \\
\hline $\begin{array}{c}\text { Edad } \\
>=65 \text { años } \\
<65 \text { años }\end{array}$ & $\begin{array}{l}428 \\
190\end{array}$ & $\begin{array}{r}69,2 \\
30,7\end{array}$ \\
\hline $\begin{array}{c}\text { Estancia preoperatoria } \\
>=2 \text { días } \\
<2 \text { días }\end{array}$ & $\begin{array}{c}563 \\
55\end{array}$ & $\begin{array}{c}91,1 \\
8,9 \\
\end{array}$ \\
\hline $\begin{array}{c}\text { Duración de la intervención* } \\
>=240 \text { minutos } \\
<240 \text { minutos }\end{array}$ & $\begin{array}{l}175 \\
436 \\
\end{array}$ & $\begin{array}{l}28,6 \\
71,4\end{array}$ \\
\hline $\begin{array}{c}\mathrm{ASA}^{*} \\
1-2 \\
3 \\
4-5\end{array}$ & $\begin{array}{l}110 \\
271 \\
130\end{array}$ & $\begin{array}{c}21,5 \\
53 \\
25,4\end{array}$ \\
\hline $\begin{array}{c}\text { NNIS* } \\
0 \\
1 \\
2-3\end{array}$ & $\begin{array}{l}100 \\
279 \\
132\end{array}$ & $\begin{array}{l}19,5 \\
54,5 \\
25,8\end{array}$ \\
\hline $\begin{array}{c}\text { Clase de intervención } \\
\text { Urgente } \\
\text { Programada }\end{array}$ & $\begin{array}{c}57 \\
561\end{array}$ & $\begin{array}{c}9,2 \\
90,8\end{array}$ \\
\hline $\begin{array}{l}\text { Diabetes* } \\
\text { Sí } \\
\text { No }\end{array}$ & $\begin{array}{l}213 \\
405\end{array}$ & $\begin{array}{l}34 \\
66\end{array}$ \\
\hline $\begin{array}{c}\text { Prótesis* } \\
\text { Dacron } \\
\text { Vena Safena } \\
\text { Homo injerto criopreservado } \\
\text { Poletetra-Fluoroetileno }\end{array}$ & $\begin{array}{c}252 \\
177 \\
28 \\
153\end{array}$ & $\begin{array}{c}41,3 \\
29 \\
4,6 \\
25\end{array}$ \\
\hline $\begin{array}{l}\text { Éxitus } \\
\text { Si } \\
\text { No }\end{array}$ & $\begin{array}{c}35 \\
583\end{array}$ & $\begin{array}{c}5,7 \\
94,3\end{array}$ \\
\hline
\end{tabular}

* pérdida de valores es esta variable.

como queda definido por el propio NNIS ${ }^{10}$; también se incluyeron las variables tipo de infección, agentes etiológicos y fecha de alta del servicio. 
Criterios de infección: Para el diagnóstico de infección quirúrgica empleamos los criterios publicados por el Center Disease Control (CDC) en el año $1992^{11}$.

Validación de los datos y análisis: Los datos recogidos se introdujeron en el programa Epiinfo versión $6.04 \mathrm{~d}^{12}$. Antes de proceder a su análisis fueron validados para identificar valores perdidos, valores extremos o valores incorrectos utilizando el mismo programa.

Para el análisis de las variable cualitativas, usamos el test de Chi Cuadrado, el test de Mantel y Haenszel, el test exacto de Fisher, y la Chi cuadrado de tendencias, y para las variables cuantitativas el test de Student.

Las variables con una $\mathrm{p}<0,1$ identificadas en el análisis univariante se incluyeron en un modelo de regresión logística para análisis multivariado. Empleamos el proceso forward stepwisse. El programa estadístico empleado fue (SPSS 10.0).

La medida de frecuencia usada fue la incidencia acumulada y de asociación el riesgo relativo (RR); junto con sus respectivos intervalos de confianza del $95 \%$.

\section{RESULTADOS:}

\section{Características generales de la población estudiada}

Desde enero de 2000 a diciembre de 2002 fueron intervenidos de by-pass en el servicio de cirugía vascular 618 pacientes. Sus características en relación a las diferentes variables se muestran en la tabla 1. Del total de pacientes estudiados a lo largo de los 3 años, 95 desarrollaron una infección de herida quirúrgica, la tasa cruda de incidencia acumulada durante este periodo de tiempo fue del $15,3 \%$ con un intervalo de confianza del 95 $\%$ comprendido entre $12,3 \%-17,9 \%$.

Desagregando por años las tasas de incidencia acumulada estas fueron: para los años 2000 a 2002 del 21,8\%, 11,6\%, y 12,7\% respectivamente. El tipo de infección presentado por los pacientes clasificadas según los criterios del CDC fue: superficial 46 $(48,4 \%)$, profunda $33(34,7 \%)$ y de órgano/espacio $16(16,8 \%)$.

Tabla 2

Microorganismos aislados en las infecciones quirúrgicas de by-pass años 2000-2002

\begin{tabular}{|l|c|c|}
\hline Microorganismo aislados & Número & $\%$ \\
\hline Staphylococcus aureus & 5 & 10 \\
\hline Staphylococcus epidermidis & 10 & 20 \\
\hline Staphylococcus aureus meticilín resistente & 6 & 12 \\
\hline Enterococcus faecalis & 4 & 8 \\
\hline Escherichia coli & 7 & 14 \\
\hline Enterobacter cloacae & 2 & 4 \\
\hline Pseudomona aeruginosa & 4 & 8 \\
\hline Proteus Indol+ & 2 & 4 \\
\hline Polimicrobiana & 7 & 14 \\
\hline Otros gérmenes & 3 & 6 \\
\hline Total & 50 & 100 \\
\hline
\end{tabular}


El intervalo diagnóstico de la infección presentó una media de 15,9 días con una desviación estándar de 17,2 días, y un rango entre 2-108 días.

Los principales microorganismos aislados causantes de las infecciones fueron Staphylococcus aureus, Staphylococcus epidermidis y Escherichia coli (tabla 2). En un 29,4\% de las infecciones no se pidieron cultivos de la herida y en un $17 \%$ estos fueron negativos.

En cuanto a la profilaxis quirúrgica (PQ) $565(91,4 \%)$ de los pacientes recibió la misma, $42(6,8 \%)$ no, y en $11(1,8 \%)$ no se encontró reflejado en la historia clínica.

Tabla 3

Análisis univariante de potenciales factores de riesgo en by-pass vascular

\begin{tabular}{|c|c|c|c|c|c|}
\hline Factor de riesgo & $\begin{array}{l}\text { Pacientes con IHQ* } \\
\text { N=95 }\end{array}$ & $\begin{array}{l}\text { Pacientes sin IHQ } \\
\qquad=523\end{array}$ & $\begin{array}{c}\text { Riesgo } \\
\text { Relativo }\end{array}$ & IC 95\% & $\mathbf{P}$ \\
\hline $\begin{array}{c}\text { Edad } \\
<65 \text { años } \\
>=65 \text { años }\end{array}$ & $\begin{array}{l}26 \\
69 \\
\end{array}$ & $\begin{array}{l}165 \\
358 \\
\end{array}$ & $\begin{array}{c}1 \\
1,2 \\
\end{array}$ & $0,8-1,8$ & NS \\
\hline $\begin{array}{c}\text { Sexo } \\
\text { Varón } \\
\text { Mujer }\end{array}$ & $\begin{array}{l}72 \\
23\end{array}$ & $\begin{array}{c}461 \\
62\end{array}$ & $\begin{array}{l}1 \\
2\end{array}$ & $1,3-3,1$ & 0,001 \\
\hline $\begin{array}{c}\text { Estancia preoperatoria } \\
<2 \text { dias } \\
>=2 \text { dias }\end{array}$ & $\begin{array}{c}9 \\
86\end{array}$ & $\begin{array}{c}46 \\
477 \\
\end{array}$ & $\begin{array}{c}1 \\
0,99\end{array}$ & $0,5-1,8$ & NS \\
\hline $\begin{array}{c}\text { Diabetes } \\
\text { No } \\
\text { Sí } \\
\end{array}$ & $\begin{array}{l}55 \\
40 \\
\end{array}$ & $\begin{array}{l}350 \\
173 \\
\end{array}$ & $\begin{array}{c}1 \\
1,4 \\
\end{array}$ & $0,97-2$ & NS \\
\hline $\begin{array}{c}\text { Cirugía urgente } \\
\text { No } \\
\text { Sí }\end{array}$ & $\begin{array}{l}85 \\
10 \\
\end{array}$ & $\begin{array}{c}476 \\
47 \\
\end{array}$ & $\begin{array}{c}1 \\
1,27 \\
\end{array}$ & $0,7-2,2$ & NS \\
\hline $\begin{array}{c}\text { Tipo de prótesis* } \\
\text { Dacron } \\
\text { Poletetra-Fluoroetileno } \\
\text { Vena safena } \\
\text { Homoinjerto criopreservado }\end{array}$ & $\begin{array}{c}29 \\
21 \\
41 \\
4\end{array}$ & $\begin{array}{c}223 \\
132 \\
136 \\
24\end{array}$ & $\begin{array}{c}1 \\
1,2 \\
2 \\
1,2\end{array}$ & $\begin{array}{c}0,7-2 \\
1,3-3,1 \\
0,5-3,2\end{array}$ & $\begin{array}{c}\text { NS } \\
0,002 \\
\text { NS }\end{array}$ \\
\hline $\begin{array}{c}\mathrm{ASA}^{*} \\
1-2 \\
>2\end{array}$ & $\begin{array}{l}17 \\
67\end{array}$ & $\begin{array}{c}93 \\
334\end{array}$ & $\begin{array}{c}1 \\
1,13\end{array}$ & $0,68-1,87$ & NS \\
\hline $\begin{array}{c}\text { Duración de la intervención* } \\
<240 \text { minutos } \\
>=240 \text { minutos }\end{array}$ & $\begin{array}{l}62 \\
33 \\
\end{array}$ & $\begin{array}{l}374 \\
142 \\
\end{array}$ & $\begin{array}{c}1 \\
1,2 \\
\end{array}$ & $0,83-1,8$ & NS \\
\hline $\begin{array}{c}\text { Indice NNIS* } \\
0 \\
1 \\
2-3\end{array}$ & $\begin{array}{l}14 \\
46 \\
24\end{array}$ & $\begin{array}{c}86 \\
233 \\
108\end{array}$ & $\begin{array}{c}1 \\
1,18 \\
1,3\end{array}$ & $\begin{array}{l}0,65-1,45 \\
0,71-2,38\end{array}$ & $\begin{array}{l}\text { NS } \\
\text { NS }\end{array}$ \\
\hline $\begin{array}{c}\text { Profilaxis quirúrgica* } \\
\text { Si } \\
\text { No }\end{array}$ & $\begin{array}{c}88 \\
7\end{array}$ & $\begin{array}{c}477 \\
35\end{array}$ & $\begin{array}{c}1 \\
1,07\end{array}$ & $0,53-2,1$ & NS \\
\hline
\end{tabular}

$\mathrm{IHQ}=$ infección de herida quirúrgica; $\mathrm{IC}=$ intervalo de confianza; $\mathrm{NS}=$ no significativo; * pérdida de valores es esta variable. 
Tabla 4

Resultados del análisis multivariante de los factores de riesgo para las infecciones quirúrgicas

\begin{tabular}{|l|l|l|l|}
\hline Factor & RR ajustado & $\mathbf{I C 9 5 \%}$ & $\mathbf{p}$ \\
\hline Sexo: mujer & 1,8 & $1,01-3,4$ & 0,043 \\
\hline Prótesis safena & 3,8 & $1,6-9,3$ & 0,02 \\
\hline ASA $>2$ & 1,7 & $1,02-29$ & 0,041 \\
\hline
\end{tabular}

$\mathrm{RR}=$ riesgo relativo $\mathrm{IC} 95 \%=$ intervalo de confianza del $95 \%$.

Tabla 5

Evolución de las tasas de incidencia acumulada a lo largo de los 3 años de estudio

\begin{tabular}{|c|c|c|c|c|}
\hline Año & I. acumulada \% & IC95\% & OR & P* \\
\hline 2000 & 21,8 & $16,5-28,1$ & 1 & 0,009 \\
\hline 2001 & 11,6 & $7,4-17$ & 0,47 & \\
\hline 2002 & 12,7 & $8,5-17,7$ & 0,52 & \\
\hline
\end{tabular}

En el $100 \%$ de los pacientes con PQ el comienzo y la duración se adecuaron a las guías de profilaxis quirúrgicas del hospital, poniéndose la primera dosis de antibiótico en la inducción anestésica. El antibiótico más utilizado fue cefuroxima $(82,9 \%)$, seguido de cefazolina $(4,3 \%)$ y clindamicina $(2,5 \%)$.

Los potenciales factores de riesgo relacionados con la adquisición de la infección que aparecen en el análisis univariado se muestran en la tabla 3. Del análisis univariante de los mismos encontramos que tan solo el sexo (mujer $\mathrm{RR}=2$ ) y la implantación de prótesis venosa del propio paciente $(R R=2)$ se asociaron con un mayor riesgo de infección quirúrgica. Tras el análisis multivariante solo identificamos como factores de riesgo el sexo, el tipo de prótesis (safena) y el ASA mayor o igual a 3. (tabla 4).

El análisis de la evolución de las tasas de incidencia de infección de herida quirúrgica a lo largo de los años 2000, 2001 y 2002 nos muestra una disminución significativa, considerando como valor basal la correspondiente al año 2000, fecha en la que iniciamos el programa de vigilancia en el servicio (tabla 5).

El exceso de estancia medido por la diferencia media en días, de la estancia postoperatoria entre pacientes infectados y no infectados fue de 8,8 días $(\mathrm{p}<0,001)$.

La incidencia de infección en función del tipo de prótesis utilizada y la localización de las infecciones nos muestra que las prótesis de dacrón y las de poletrafluoretileno presentan las tasas más elevadas de infección de órgano $(2,8 \%$ y $2,6 \%$ respectivamente), mientras que los injertos de vena safena tienen una tasa para la misma clasificación del $1,7 \%$, ocurriendo lo inverso con las infecciones de localización superficial: 12,9\% para safena, y $4,7 \%$ y $7,1 \%$ para dacron y poletetrafluoretileno.

\section{DISCUSIÓN}

Las infecciones quirúrgicas en pacientes intervenidos para la implantación de by-pass suponen un problema importante para la 
cirugía vascular por las complicaciones que pueden derivarse de las mismas, presentando una tasa de mortalidad que oscila entre un $15-25 \%$ y hasta un $40 \%$ de miembros amputados cuando las infecciones tienen lugar a nivel de la prótesis ${ }^{13}$.

La incidencia de las infecciones presenta un rango que va desde el 1\% al 44\%14,15. Esta amplia variabilidad puede ser debida a la utilización de criterios diferentes de identificación de las infecciones o a la no diferenciación de los procedimientos quirúrgicos. Los resultados encontrados en nuestro estudio aplicando los criterios del $\mathrm{CDC}^{11}$ muestran una tasa cruda de $15,3 \%$, con un rango entre $22,6 \%$ y $11,2 \%$, lo que está en consonancia con los datos publicados por otros autores utilizando criterios de clasificación de las infecciones diferentes ${ }^{14-16}$ con tasas que oscilan entre el $11 \%$ y el $19 \%$. Si desagregamos por tipo de infección encontramos que la tasa de infección protésica (la clasificada como infección de órgano) ${ }^{11}$ es del $2,5 \%$, semejante a las notificadas por otros autores entre un $2 \%$ a $4 \%$ respectivamente ${ }^{16-}$ 18 . En cuanto a los microorganismos causantes de las infecciones la mayoría de los estudios publicados ponen de manifiesto una mayor incidencia de gram positivos. Staphylococcus epidermidis, Staphylococcus aureus y Enterococcus faecalis, seguidos de gram negativos, fundamentalmente enterobacterias ${ }^{19,20}$. En nuestro estudio los microorganismos más frecuentes coinciden con los anteriores, pero con una mayor incidencia de Staphylococcus aureus y dentro de éste destaca la importancia de Staphylococcus aureus meticilin resistente que representa más del 50\% de los Staphilococcus aureus aislados. El análisis de los potenciales factores de riesgo pone de manifiesto que las mujeres tienen un mayor riesgo de desarrollar infección de la herida quirúrgica, tal y como también pusieron de manifiesto Belkin et $\mathrm{al}^{21}$ en cuyo estudio el sexo femenino presenta una mayor incidencia de infección ( $13,5 \%$ frente a $3,3 \%$ de los varones), aunque las mujeres tienen una mortalidad menor. El tipo de prótesis implantada es otro de los factores de riesgo identificados, presentando mayor incidencia de infección los pacientes a los que se les implantó una prótesis de vena safena, hecho puesto de manifiesto por Chang et al. ${ }^{22}$. Estos autores encuentran una mayor frecuencia de infecciones superficiales, mientras que las infecciones más graves, las de órgano, inciden en las prótesis de tetrafluoroetileno, lo que concuerda plenamente con nuestros resultados, ya que las infecciones más frecuentes con el uso de vena safena eran las superficiales con un $12,9 \%$; mientras que las infecciones de órgano presentaban una mayor tasa de infección con las prótesis de dacron $2,8 \%$, seguidas de tetrafluoroetileno con un 2,6\%. Esto podría explicarse por el hecho de que las prótesis de dacron y de tetrafluoroetileno se comportan como cuerpos extraños, lo que favorecería la infección interna de las mismas. No encontramos en el análisis ninguna otra diferencia que explique la mayor incidencia de infecciones más graves en estos pacientes. El tercer factor de riesgo encontrado fue el índice de riesgo ASA mayor de 2. Otros potenciales factores de riesgo como la diabetes no se pusieron de manifiesto ni en el análisis univariado ni en la regresión logística. Aunque este factor ha sido implicado en diferentes publicaciones como responsable del aumento de la infección en pacientes quirúrgicos, y concretamente en pacientes de cirugía vascular, otros estudios no encuentran dicha asociación ${ }^{22}$.

A diferencia de otros autores no encontramos que la duración de la intervención sea un factor de riesgo, ya que el percentil 75 de la duración en minutos es el mismo, tanto para los pacientes con infección como los que no la presentan.

La profilaxis quirúrgica se considera un factor protector de la infección cuando se aplica adecuadamente. En nuestro estudio el $91,8 \%$ de los pacientes había recibido profilaxis antibiótica de forma correcta, no encontrando diferencias entre infectados y no infectados. 
En cuanto a la estancia postoperatoria encontramos una diferencia importante entre los pacientes infectados y los no infectados, estimada en un exceso de hospitalización entre los pacientes con infección de 8,8 días de media.

Aunque no era un objetivo del estudio evaluar la eficacia del programa en la reducción de las tasas de infección, la disminución encontrada a lo largo de los 2 años posteriores puede ser debida a la información suministrada a los cirujanos y al intercambio de información con el personal de la unidad llevado a cabo a lo largo del estudio.

Como conclusiones de nuestro estudio destacamos la identificación de tres factores de riesgo, aunque la capacidad de influencia sobre alguno de ellos sea prácticamente nula. La identificación de la prótesis de vena safena como la que causa el mayor número de infecciones, aunque la mayoría son superficiales, conocer la incidencia de infección quirúrgica de by-pass vasculares en nuestro hospital, así como los microorganismos que más frecuentemente causan las mismas.

\section{BIBLIOGRAFÍA}

1. Calligaro KD, Veith FJ, Swartz ML, Dougherty MJ, DeLaurentis DA. Differences in early versus late extracavitary arterial graft infections. J Vasc Surg 1995; 22:680-8.

2. Calligaro KD, Veith FJ, Swartz ML, Goldsmith J, Savarese RP, Dougherty MJ, DeLaurentis DA. Selective preservation of infected prosthetic arterial grafts analysis of a 20 years experience with 120 extracavitary-infected grafts. Ann Surg 1994; 220: 461-71.

3. Bandy DF. Infection in prosthetic vascular graft. En Rutherford R B, editores. Rutherford Vascular Surgery. $5^{\mathrm{a}}$ ed. Vol 1 cap 47. WB Saunders; 2000 p. 733-51.

4. Goldstone J, Malone JM, Mcintyre KE. Infections associated with prosthetic arterial grafts. En Sugarman B, Young EJ, editors. Infections Associated with Prostehtic Devices. Boca Raton FL:CRC Press 1984. p.123-41.
5. R J Segura Iglesias. Reflexiones sobre la infección protésica. Angiología 2002; 54:61-63.

6. Sobregrau RC, Callejas JM, Collado LM, Ortiz E, Rodríguez A. Infección de los injertos de Dacron. Angiología 1981; 33:328-40.

7. Clagett GP, Valentine RJ, Hagino RT. Autogenous aortoiliac/femoral reconstruction from superficial femoral-popliteal veins:feasibility and durability. J Vasc Surg 1997;25:255-77.

8. Keats A S. The ASA classification of physical status a recapitulation. Anesthesiology 1978;49:233-

9. Altemeier WA, Burke JF, Pruitt BA, Sandusky WR editores. Manual on control of infections in surgical patients. Philadephia: JB Lippincott;1992.

10 National Nosocomial Infections Surveillance (NNIS) System report, data summary from January 1990-may 1999 issued june 1999. Am J Infect Control 1999;27:520-32.

11 Horan TC, Gaynes RP, Martone WJ, Jarvis WR, Emory TG. CDC definitions of nosocomial surgical infections, 1992: a modification of CDC definitions of surgical wound infections. Am J Infect Control 1992;20:271-4.

12 Dean A.G.,Dean J.A., Coulombier D. et al. Epi info, version 6: A word processing, database, and statistics program for epidemiology on microcomputers. Georgia: Center for Disease Control and Prevention Atlanta; 1994

13 Rodríguez A, Royo J, Bofia R, Escribano JA, Sancho J, Samso M et al. Tratamiento quirúrgico de las infecciones de las prótesis arteriales del sector aorto-iliaco: una década de experiencia. Angiología 1996;48:61-7.

14 Nam JH, Gatham V, Roberts AB, and Kerstein MD. Influence of Incisional complications on Infrainguinal Vein Bypass Graft Outcome. Ann Vas Surg 1999;13: 77-83.

15 Lee ES, Santilli SM, Olsosn MM, Kuskowski MA Lee TJ. Wound infections after infrainfuinal bypass operations:multivariate análisis of putative risk factors. Surg Infect 2000; 4 : 257-63.

16 Monreal M, Callejas JM, Lisbona C, Martorell A, Lerma R, Boabaid R, Mejia S. Infección de herida quirúrgica en pacientes sometidos a cirugía arterial extraanatómica. Un estudio retrospectivo. Angiología 1993; 45: 199-202. 
17 Bonelli U, Cerruti R, Baglieto F, Cantello C, Znicola $\mathrm{N}$. Incidence and treatment of vascular prosthesis infection in the groin. Personal experience. Minerva Chir.1994; 49: 807-11.

18 JL Cabrera Zamora, A Hernández Seara, P .González Amaro y C Duran Lobera. Infección protésica en los servicios de cirugía vascular. Rev Cubana Angiol y Cir Vasc 2001; 2: 5-9.

19 Jhonson JA, Corbgill TH, Strutt PJ, and Gundersen. All Wound Complications after infrainguinal Bypass. Arch Surg 1998; 123: 859-62.
20 Van Himbeeck FJ, van Knippenberg LA, Niessen MC,van Griethuys AJ. Wound infection after arterial surgical procedures. Eur J Vasc Surg 1992; 6: 494-8.

21 Belkin M, Conte MS, Donaldson MC, Mannick JA, Whitermore AD. The impact of gender on the results of arterial bypass with in situ greater saphenous vein. Am J Surg 1995;170: 97-102.

22 Chang JK, Calligaro KD, Ryan S, Runyan D, Dougherty MJ.Stern J. Risk factors associated with infection of lower extremity revascularization: Analysis of 365 procedures performed at teaching hospital. Ann Vasc Surg 2003; 17: 91-6. 